\title{
Spleen enlargement assessment using computed tomography: which coefficient correlates the strongest with the real volume of the spleen?
}

\author{
Iwona Kucybała๑, ${ }^{1}$ Szymon Ciuk, ${ }^{2}$ Justyna Tęczar ${ }^{1}$ \\ ${ }^{1}$ Department of Radiology, Jagiellonian University Medical College, 19 Kopernika Street, 31-501 Kraków, Poland \\ ${ }^{2}$ Department of General and Interventional Radiology and Neuroradiology, Wroclaw Medical University, 213 Borowska Street, \\ 50-556 Wrocaw, Poland
}

\section{Abstract}

Purpose: The aim of the study was to find which linear measurements, field and volume coefficients correlate the best with real volume of the spleen and can be further used for identification of splenomegaly.

Methods: Abdominal computed tomography (CT) examinations of 264 patients were retrospectively analyzed in terms of maximal length, maximal thickness, hilum thickness, maximal height, vertical height and estimated height. Spleen volume was manually measured in Vitrea software. Two- and three-dimensional coefficients were calculated through proper mathematical formulas from linear measurements. Splenomegaly cut-off: $314.5 \mathrm{~cm}^{3}$. Data were analyzed with use of Pearson correlation and $\chi^{2}$ test with statistical significance at $p<0.05$.

Results: For single measurements, the correlation with real spleen volume was the strongest for maximal height $(r=0.804 ; p<0.05)$. Among two-dimensional indexes, multiplication product of maximal length and vertical height reached the highest level of correlation with spleen volume $(r=0.923 ; p<0.05)$ and had the highest sensitivity and specificity $(94.3 \%$ and $93.0 \%$, respectively) for splenomegaly detection (threshold $115 \mathrm{~cm}^{2}$ ). In case of three-dimensional ones, the coefficient calculated from maximal length, vertical height and hilum thickness established the strongest link with spleen volume $(r=0.956 ; p<0.05)$.

Conclusions: Coefficient calculated from maximal length, vertical height and hilum thickness correlates the strongest with spleen volume and can be utilized for

Correspondence to: Iwona Kucybała; email: iwona.kucybala@gmail. com monitoring of spleen volume instead of obsolete splenic index. The most suitable for quick splenomegaly screening is two-dimensional coefficient (maximal length $\times$ vertical height), with the cut-off $115 \mathrm{~cm}^{2}$.

Key words: Spleen-Splenomegaly_-Spleen volume - Splenic index-Computed tomography

Splenomegaly is a common clinical pathological finding of various aetiologies, including congestive, excessive antigenic stimulation, destruction of abnormal blood cells and neoplastic infiltration. It may be detected in symptomatic and coincidentally, in asymptomatic patients. Thus, appropriate evaluation of the spleen size seems to be necessary in order to initiate a diagnostic process, take proper therapeutic decisions and follow treatment effect in definite cases $[1,2]$.

Primarily, the clinical diagnosis of splenomegaly is defined as the organ palpable below the costal margin during physical examination [3]. However, the recognition of the spleen enlargement by means of imaging methods such as ultrasonography or computed tomography was proved to be more sensitive [4]. Currently, computed tomography (CT) is considered as a highly accurate and reproducible diagnostic tool for spleen morphology assessment, since it allows to thoroughly evaluate the spleen on multiple intersections, and is widely available and relatively cheap [5].

There have been multiple approaches for a precise evaluation of spleen size basing on CT examination. To this end, individual linear measurements, multidimensional coefficients as well as volume measurements have 
been applied. These include a popular, but rather obsolete formula called splenic index-multiplication product of maximal length, estimated height and maximal thickness of the spleen with the upper normality limit for splenomegaly set at 480 [6].

Clinical routine requires simultaneously reliable and practical solutions. At present, clear and unequivocal guidelines for splenomegaly assessment in CT are lacking. Therefore, the aim of this study was to find which linear measurements and two-dimensional and three-dimensional coefficients correlate the best with real volume of the spleen and can be further used for determination of splenomegaly.

\section{Materials and methods}

\section{Study population}

We included into the study and retrospectively analyzed 264 abdominal CT examinations, which were carried out at the Department of Diagnostic Imaging of the University Hospital (Szpital Uniwersytecki) in Cracow in the period between September and December 2016. Mean age of examined patients was $58.8 \pm 15.5$ years, age ranged between 16 and 91 years. $48.5 \%(n=128)$ of the patients were female and $51.5 \%(n=136)$ male. Patients included into the study were referred to the Department of Diagnostic Imaging to undergo abdominal CT due to various clinical symptoms related to the abdominal region. The exclusion criteria were spleen injury, multi-organ injury, spleen focal lesion, previous splenectomy and any condition that would affect the position of the spleen.

\section{Technical parameters}

All of the scans were performed with use of an 80-row helical CT scanner Toshiba Aquilion PRIME 80 (Toshiba America Medical System, Irvine, CA), in an abdominal protocol, scan parameters: $120 \mathrm{kV}, 385 \mathrm{mAs}$; rotation time $500 \mathrm{~ms}$; slice thickness $2.0 \mathrm{~mm}$, interval $1.6 \mathrm{~mm}$, to acquire axial images of the abdominal region. Coronal sections were obtained using multiplanar reconstructions (iterative reconstruction method), parameters: slice thickness $2.0 \mathrm{~mm}$, interval $2.0 \mathrm{~mm}$.

\section{Measurements methodology}

Linear measurements and volume of the spleen parenchyma $\left(V_{\mathrm{spl}}\right)$ were assessed with the use of Vitrea Software 6.7.1 (Vital Images Inc., Minnetonka, MN, USA). All linear measurements were done in millimeters, while volume of the spleen was calculated in $\mathrm{cm}^{3}$. All values of measurements were presented as the value \pm standard deviation.

Linear dimensions of the spleen measured in the axial section were (Fig. 1):

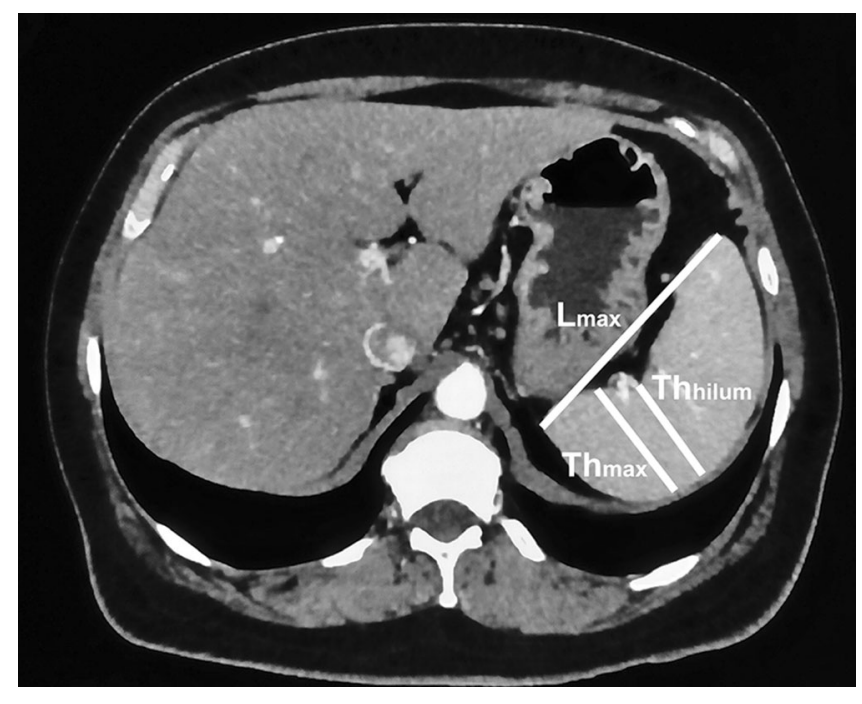

Fig. 1. The method for measuring of maximal length $\left(L_{\max }\right)$, maximal thickness $\left(\mathrm{Th}_{\max }\right)$ and hilum thickness $\left(\mathrm{Th}_{\text {hilum }}\right)$.

- maximal length ( $\left.L_{\max }\right)$ - the longest dimension between poles of the spleen in this section,

- maximal thickness $\left(\mathrm{Th}_{\max }\right)$ - the widest dimension perpendicular to the long axis of the spleen,

- hilum thickness $\left(\mathrm{Th}_{\text {hilum }}\right)$-dimension measured in central part of the hilum perpendicularly to the long axis of the spleen.

In the coronal section (Fig. 2):

- maximal height $\left(H_{\max }\right)$ - the longest dimension between poles of the spleen in this section,

- vertical height $\left(H_{\text {vert }}\right)$ - the longest vertical dimension between cranial and caudal borders of the spleen.

Additionally, estimated height of the spleen $\left(H_{\text {est }}\right)$ was assessed as the number of axial scans with the spleen visible multiplied by the slice thickness.

Two-dimensional (2D) and three-dimensional (3D) coefficients were calculated as the product of multipli-

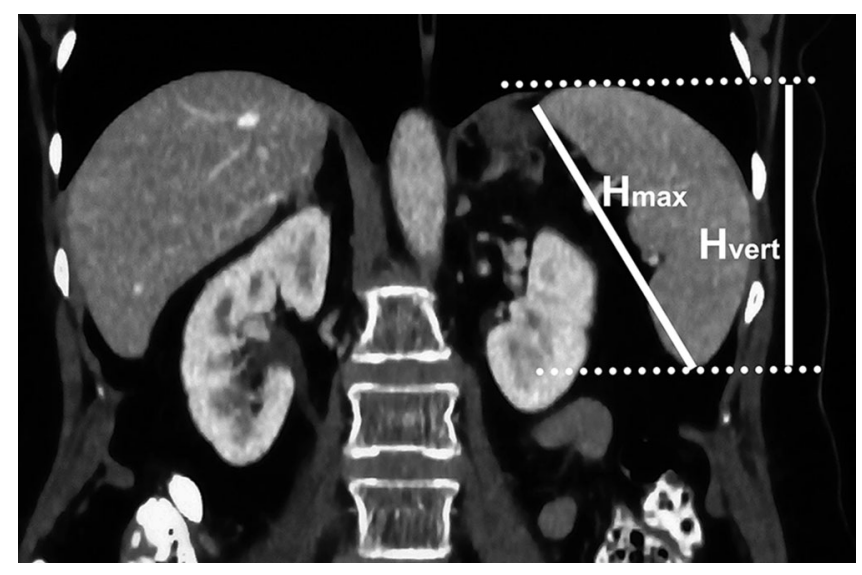

Fig. 2. Dimensions measured on the coronal plane-maximal height $\left(H_{\max }\right)$ and vertical height $\left(H_{\text {vert }}\right)$. 
cation of respectively two (length $\times$ thickness, length $\times$ height, height $\times$ thickness) or three various dimensions (length $\times$ thickness $\times$ height). In our study, 11 two-dimensional and 6 three-dimensional coefficients were counted.

The real spleen volume was measured manually with the Volume Measurement Tool by defining the frontiers of the spleen on the surface of fibrous capsule (in a soft tissue window) in the coronal section. Subsequently, the volume was calculated by the software and three-dimensional reconstruction of the organ was obtained (Fig. 3).

All measurements of the spleen were evaluated by two independent examiners simultaneously and the mean of two separate measurements of the identical parameter was classified as the final value. All significant discrepancies in measurements between examiners were analyzed and assessed one more time in the presence of a third examiner.

\section{Statistical analysis}

Statistical analysis was performed in Statistica 12.5 software (StatSoft Inc., Tulsa, OK, USA). Statistical significance was set at $p<0.05$. Linear Pearson's correlation was used to assess the strength of correlation between dimensions along with coefficients and volume of the spleen.

Cut-off points for detection of splenomegaly for linear measurements along with two-dimensional and threedimensional coefficients were calculated in each case from equations of linear regression calculated by the software. Value of $314.5 \mathrm{~cm}^{3}$ as volumetric threshold of splenomegaly, according to Prassopoulos et al. study [6], was substituted into specific linear regression equation and the product of this calculation was considered as the splenomegaly cut-off value of certain parameter. In cases of linear dimensions, all results were rounded up or down to the nearest half while products of two-dimensional and three-dimensional coefficients were rounded up or down to the nearest integer. In the next step, Chi square

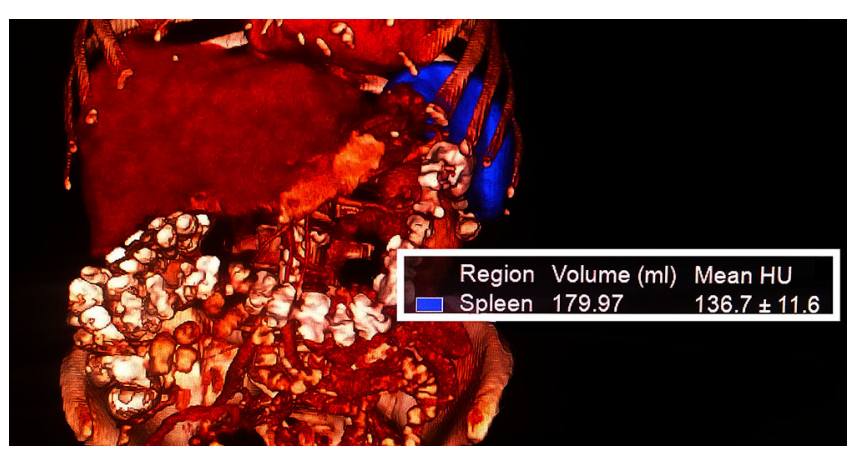

Fig. 3. Three-dimensional reconstruction of the spleen and the calculation of its volume. test, sensitivity, specificity, positive predictive value (PPV) and negative predictive value (NPV) were applied so as to evaluate the capability of calculated threshold to detect splenomegaly according to volumetric criterion and to assess its effectiveness.

\section{Results}

$13.3 \%(n=35)$ of patients in the study had splenomegaly according to previously mentioned volumetric criterion. Reasons of spleen enlargement in our study were liver cirrhosis, Hodgkin lymphoma, chronic lymphoid leukemia (CLL), hemolytic anemia and acquired immune deficiency syndrome (AIDS). Mean volume of the spleen were $178.1 \pm 64.2$ and $481.4 \pm 242.0 \mathrm{~cm}^{3}$ in groups without and with splenomegaly, respectively. Any statistically significant differences in terms of age and gender between groups with or without splenomegaly were not detected (Table 1).

\section{Linear measurements}

The strongest correlation with splenic volume was detected for maximal height $(r=0.804 ; p<0.05)$. Splenomegaly threshold for this parameter was $12 \mathrm{~cm}$ (equation of linear regression: $V_{\mathrm{spl}}=6.393 \times H_{\max }+$ 444.129). Sensitivity, specificity, PPV and NPV values were $71.4,91.7,56.8$ and $95.5 \%$.

Similar strength of correlation was observed in case of vertical height $(r=0.790 ; p<0.05)$. Calculated splenomegaly cut-off was $10.5 \mathrm{~cm}$ (equation: $V_{\mathrm{spl}}=4.986 \times$ $\left.H_{\text {vert }}+210,541\right)$. This dimension improved values of sensitivity, specificity, PPV and NPV: 82.9, 91.7, 60.4 and $97.2 \%$, respectively.

Profile of all linear dimensions is shown in Table 2.

\section{Two-dimensional coefficients}

Multiplication product of maximal length and vertical height correlated the strongest with volume of the spleen $\left(r=0.923 ; \quad p<0.05\right.$; equation: $V_{\text {spl }}=3.514 \times$ $\left.\left(L_{\max } \times H_{\mathrm{vert}}\right)-92.464\right)$. Calculated splenomegaly threshold: $116 \mathrm{~cm}^{2}$. Approximation to $115 \mathrm{~cm}^{2}$ did not have any impact on qualitative parameters of effectiveness of the coefficient. Sensitivity, specificity, PPV and NPV values for 115 and $116 \mathrm{~cm}^{2}$ were 94.3, 93.0, 67.3 and $99.1 \%$, respectively. Rounding up to $120 \mathrm{~cm}^{2}$ caused a slight decline in the value of sensitivity (91.4\%) and NPV (98.6\%) still remaining at a high level and elevation of specificity (94.3\%) and PPV (71.1\%).

Table 1. Demographic data of groups with or without splenomegaly

\begin{tabular}{lllr}
\hline & Splenomegaly & Without splenomegaly & $p$ value \\
\hline Age \pm SD (years) & $55.2 \pm 12.6$ & $59.3 \pm 15.1$ & 0.120 \\
Females (\%) & 40.0 & 49.8 & 0.281 \\
\hline
\end{tabular}


Table 2. Correlation between linear dimensions and volume of the spleen; calculated splenomegaly cut-offs and their effectiveness in detection of volumetric splenomegaly; in bold - best correlation

\begin{tabular}{|c|c|c|c|c|c|c|c|c|}
\hline \multirow[t]{2}{*}{ Linear dimension } & \multicolumn{2}{|c|}{$\begin{array}{l}\text { Pearson linear cor- } \\
\text { relation }\end{array}$} & \multirow[t]{2}{*}{$\begin{array}{l}\text { Splenomegaly } \\
\text { cut-off }(\mathrm{cm})\end{array}$} & \multirow[t]{2}{*}{$p$ value } & \multirow[t]{2}{*}{ Sensitivity (\%) } & \multirow[t]{2}{*}{ Specificity (\%) } & \multirow[t]{2}{*}{ PPV $(\%)$} & \multirow[t]{2}{*}{ NPV $(\%)$} \\
\hline & $p$ value & $r$ & & & & & & \\
\hline Maximal length & $p<0.05$ & 0.756 & 11.5 & $p<0.05$ & 80.0 & 90.8 & 57.1 & 96.7 \\
\hline Maximal thickness & & 0.585 & 7 & & 37.1 & 90.4 & 37.1 & 90.4 \\
\hline Hilum thickness & & 0.649 & 4 & & 68.6 & 76.0 & 30.4 & 94.1 \\
\hline Maximal height & & 0.804 & 12 & & 71.4 & 91.7 & 56.8 & 95.5 \\
\hline Vertical height & & 0.790 & 10.5 & & 82.9 & 91.7 & 60.4 & 97.2 \\
\hline Estimated height & & 0.563 & 15.5 & & 40.0 & 91.3 & 41.2 & 90.9 \\
\hline
\end{tabular}

Table 3. Correlation between two-dimensional coefficients and volume of the spleen along with splenomegaly thresholds and their effectiveness in detection of volumetric splenomegaly; in bold - best correlation

\begin{tabular}{|c|c|c|c|c|c|c|c|}
\hline \multicolumn{2}{|c|}{ Multiplied dimensions } & \multicolumn{2}{|c|}{$\begin{array}{c}\text { Pearson linear corre- } \\
\text { lation }\end{array}$} & \multirow[t]{2}{*}{$\begin{array}{l}\text { Splenomegaly } \\
\text { cut-off }\left(\mathrm{cm}^{2}\right)\end{array}$} & \multirow[t]{2}{*}{$\begin{array}{c}\chi^{2} \\
p \text { value }\end{array}$} & \multirow[t]{2}{*}{ Sensitivity (\%) } & \multirow[t]{2}{*}{ Specificity } \\
\hline Dimension 1 & Dimension 2 & $p$ value & $r$ & & & & \\
\hline Maximal length & Maximal thickness & $p<0.05$ & 0.816 & 72 & $p<0.05$ & 65.7 & 92.6 \\
\hline Maximal length & Hilum thickness & & 0.853 & 47 & & 68.6 & 90.8 \\
\hline Maximal length & Maximal height & & 0.912 & 132 & & 85.7 & 90.4 \\
\hline Maximal height & Maximal thickness & & 0.821 & 75 & & 65.7 & 90.0 \\
\hline Maximal height & Hilum thickness & & 0.851 & 49 & & 65.7 & 90.4 \\
\hline \multirow[t]{3}{*}{ Maximal length } & Vertical height & & 0.923 & 115 & & 94.3 & 93.0 \\
\hline & & & & 116 & & 94.3 & 93.0 \\
\hline & & & & 120 & & 91.4 & 94.3 \\
\hline Maximal length & Estimated height & & 0.766 & 161 & & 71.4 & 93.9 \\
\hline Maximal thickness & Vertical height & & 0.864 & 65 & & 68.6 & 93.9 \\
\hline Maximal thickness & Estimated height & & 0.726 & 91 & & 54.3 & 92.1 \\
\hline Hilum thickness & Vertical height & & 0.885 & 43 & & 71.4 & 92.1 \\
\hline Hilum thickness & Estimated height & & 0.757 & 59 & & 54.3 & 92.6 \\
\hline
\end{tabular}

Details regarding all two-dimensional coefficients are demonstrated in Table 3.

\section{Three-dimensional coefficients}

The strongest correlation with the spleen volume was discovered for a coefficient calculated by multiplication of maximal length, vertical height and hilum thickness $\left(r=0.956 ; p<0.05\right.$; linear regression equation: $V_{\mathrm{spl}}=$ $\left.0.601 \times\left(L_{\max } \times H_{\text {vert }} \times \mathrm{Th}_{\text {hilum }}\right)+18.889\right)$. The cutoff point for splenomegaly was $492 \mathrm{~cm}^{3}$. Values of sensitivity, specificity, PPV and NPV were equal for $492 \mathrm{~cm}^{3}$ and approximated $490 \mathrm{~cm}^{3}: 82.9 \%, 95.6 \%, 74.4 \%$ and $97.3 \%$, respectively. Rounding up to $500 \mathrm{~cm}^{3}$ had a negative impact on specificity (77.1\%) and NPV (96.5\%), but slightly increased the level of specificity $(96.5 \%)$ and PPV (77.1\%).

A coefficient counted by multiplication of maximal length, vertical height and maximal thickness had a fine correlation with the spleen volume as well $(r=0.946$; $p<0.05$; equation: $\quad V_{\text {spl }}=0.403 \times\left(L_{\max } \times H_{\text {vert }} \times\right.$ $\left.\left(\mathrm{Th}_{\max }\right)+18.889\right)$. Additionally, for the threshold at the level of $739 \mathrm{~cm}^{3}$, it had improved values of specificity (96.1\%) and PPV (76.3\%) along with equal sensitivity and NPV to the above-mentioned coefficient. Nonetheless, approximation to $740 \mathrm{~cm}^{3}$ alters sensitivity, specificity,
PPV and NPV to values $80 \%, 96.5 \%, 77.8 \%$ and $96.9 \%$, respectively.

The correlation of splenic index (product of multiplication of maximal length, estimated height and maximal thickness) with volume of the spleen was rather poor $\quad\left(r=0.846 ; \quad p<0.05\right.$; equation: $\quad V_{\text {spl }}=$ $\left.0.256 \times\left(L_{\max } \times H_{\text {est }} \times \mathrm{Th}_{\max }\right)+56.023\right)$, compared to other three-dimensional coefficients. The calculated cutoff point for that coefficient was $1010 \mathrm{~cm}^{3}$. Sensitivity, specificity, PPV and NPV were 62.9, 93.9, 61.1 and $94.3 \%$, respectively.

A detailed list of all parameters of three-dimensional coefficients is included in Table 4.

\section{Discussion}

The use of imaging techniques is recently considered to be the most accurate method of both diagnosis and follow-up in the case of splenomegaly. Literature suggests that CT should identify changing spleen volume with the highest sensitivity and specificity [7], although other imaging techniques such as ultrasonography [8,9] or magnetic resonance imaging (MRI) [10] can also be used. Additionally, a number of studies proved significant accuracy of the CT in assessment of the spleen, both in pediatric and adult populations $[11,12]$. 
Table 4. Correlation between three-dimensional coefficients and volume of the spleen; calculated splenomegaly cut-offs and their effectiveness in detection of volumetric splenomegaly; in bold — best correlation and in italics - splenic index

\begin{tabular}{|c|c|c|c|c|c|c|c|c|}
\hline \multicolumn{3}{|c|}{ Multiplied dimensions } & \multicolumn{2}{|c|}{$\begin{array}{l}\text { Pearson linear } \\
\text { correlation }\end{array}$} & \multirow[t]{2}{*}{$\begin{array}{l}\text { Splenomegaly } \\
\text { cut-off }(\mathrm{cm})\end{array}$} & \multirow[t]{2}{*}{$p$ value } & \multirow[t]{2}{*}{ Sensitivity $(\%)$} & \multirow[t]{2}{*}{ Specificity } \\
\hline Dimension 1 & Dimension 2 & Dimension 3 & $p$ value & $r$ & & & & \\
\hline Maximal length & Maximal height & Maximal thickness & $p<0.05$ & 0.926 & 840 & $p<0.05$ & 82.9 & 93.0 \\
\hline Maximal length & Maximal height & Hilum thickness & & 0.940 & 557 & & 68.6 & 94.8 \\
\hline \multirow[t]{2}{*}{ Maximal length } & Vertical height & Maximal thickness & & 0.946 & 739 & & 82.9 & 96.1 \\
\hline & & & & & 740 & & 80.0 & 96.5 \\
\hline \multirow{3}{*}{ Maximal length } & Vertical height & Hilum thickness & & 0.956 & 490 & & 82.9 & 95.6 \\
\hline & & & & & 492 & & 82.9 & 95.6 \\
\hline & & & & & 500 & & 77.1 & 96.5 \\
\hline Maximal length & Estimated height & Maximal thickness & & 0.846 & 1010 & & 62.9 & 93.9 \\
\hline Maximal length & Estimated height & Hilum thickness & & 0.873 & 660 & & 62.9 & 94.3 \\
\hline
\end{tabular}

Among all coefficients, three-dimensional ones seem to be the best means of spleen size evaluation, since they represent the highest level of correlation with real volume of the spleen. The highest correlation coefficient was detected for the multiplication product of maximal length, vertical height and hilum thickness of the spleen $(r=0.956 ; p<0.05)$. Moreover, the coefficient calculated from maximal length, vertical height and maximal thickness had a fine correlation with the spleen volume as well $(r=0.946 ; p<0.05)$. Thus, they can be valuable for the assessment of changes in the spleen volume in cases in which this parameter is helpful for stating the diagnosis (sarcoidosis, rheumatoid arthritis, lupus, conditions ongoing with portal hypertension), or monitoring the clinical condition, e.g., after spleen embolization, during the treatment of hemolytic anemia, myeloproliferative disorders, lymphoproliferative syndromes, acute leukemia, storage disorders (like Gaucher's or Neumann-Pick's disease) [13]. In contrast to above-mentioned coefficients, splenic index happened to have a rather weak correlation with real spleen volume $(r=0.846 ; p<0.05)$. Lackner et al. introduced the term of splenic index defined as a multiplication product of maximal length, estimated height and maximal thickness of the spleen. Comparing its value set with the use of computed tomography with the one assessed during the autopsy, it was observed that CT have a tendency to underestimate and overestimate this index. As for its limitations, a study was carried out on a relatively small group (118 patients) in comparison to ours (264 patients). Additionally, Lackner et al. study was carried out in the times when CT quality was rather poor in comparison to the technology at present, which could have affected the accuracy of measurements. However, age, race and gender differences in spleen volume were not taken into account in both studies [14]. Since the splenic index is a useful tool for instance in diagnostics of the Hodgkin lymphoma [2] and makes the sensitivity of diagnosis higher, it would be vital to update the coefficient for more clinical accuracy. The threshold indicating splenomegaly calculated for the coefficient with the strongest correlation with spleen volume (maximal length $\times$ vertical height $\times$ hilum thickness) in our study was very similar to the one suggested in the original study (490 vs. $480 \mathrm{~cm}^{3}$, respectively). Nevertheless, the cut-off point for the splenic index calculated in our study $\left(1010 \mathrm{~cm}^{3}\right)$ differs considerably from the one suggested by Lackner et al., which makes their coefficient totally unreliable to use.

The two-dimensional coefficient, set as a result of multiplying maximal length and vertical height, represented a high level of correlation with the real volume of the spleen $(r=0.923 ; p<0.05)$. Moreover, it happened to present the highest sensitivity and specificity $(94.3 \%$ and $93.0 \%$, respectively), which makes it a great screening tool for radiologists for the quick evaluation of the spleen. With a threshold indicating splenomegaly set at the level of $115 \mathrm{~cm}^{2}$, it would help to give a hint for clinicians if they should take a closer look on the spleen and supposedly pay more attention to this organ, and generally, conditions connected with spleen enlargement in terms of the future diagnostic process. Since this coefficient is a combination of measurements done in two sections - axial and coronal, it lowers the chance to overlook the spleen enlargement, in comparison to twodimensional coefficients based on dimensions measured only in one section. The splenomegaly threshold can be rounded up to $120 \mathrm{~cm}^{2}$ (to be easily memorized). Despite still high level of specificity (94.3\%), this approximation leads to a decline in sensitivity (91.4\%) and consequently, it needs to be considered if such changes are worth establishing.

Considering the linear measurements, maximal height $(r=0.804 ; p<0.05)$ of the spleen represented the strongest correlation with the volume. This measurement is consistent with the so-called cephalocaudal spleen height, which is used in clinical practice for a quick assessment of the spleen size in CT [15]. Bezerra et al. suggested length (maximal length in our study) and width (our maximal thickness) as the good indicators of 
spleen enlargement, but they both did not represent the strongest of correlation with the volume $(r=0.756$ and $r=0.585$, respectively) [16]. Maximal height parameter is consistent with craniocaudal length of the spleen measured in the ultrasound technique and so is the threshold suggesting splenomegaly for both tools $-12 \mathrm{~cm}$ [17]. It needs to be remembered that these unidimensional measurements are characterized with a few shortcomings, which derive from the different morphology or localization of the spleen. Moreover, spleen might enlarge in multiple directions, sometimes irregularly and therefore this method of assessment should be reserved for just a quick overview of this organ. However, it is still the least time-consuming method of a brief evaluation.

There are also more complex methods of defining splenomegaly, such as the relationship of the spleen to the left lobe of the liver (ultrasound) [18], or to the left kidney (CT) [16], but they are of no statistical significance or low sensitivity, respectively. Additionally, measurements done with the use of ultrasound are characterized with a low level of repeatability and depend much on the knowledge and experience of a radiologist performing the examination. The shape and spatial orientation of the spleen make it difficult to capture accurate linear measurement and to assess all three dimensions for the most reliable volume calculation. Yetter et al. invented a complicated formula, which includes width, thickness, maximum length and craniocaudal length, which correlates the best to the CT measurements [11]. However, that method of assessment is extremely intricate, requires significant amount of effort and was not useful in clinical practice. Furthermore, Chen et al. suggested the use of MRI in the assessment of spleen volume (in diagnosis of the level of liver cirrhosis) should be reliable, but, in fact, it is a more expensive and time-consuming tool [10]. Due to the multiplicity of sequences, this method should stay reserved for a detailed diagnosis of focal or diffuse lesions, which might look similar in the CT examination [19].

The spleen volume is directly linked with the weight of the organ. It has been known for a long time that spleen weight increases proportionally to the body weight, declines with the age and is generally slightly smaller among women [20]. Thus, for a detailed and thorough examination of the spleen, different thresholds should be established for groups divided according to age, sex and BMI. As well, the number of our patients with splenomegaly could also be the limitation; therefore, a larger, preferably multicenter, study should be also conducted.

It needs to be remembered that the definition of splenomegaly is highly individual and its diagnosis should always be tightly bound with a clinical condition of the patient, rather than treating it as an isolated radiologic diagnosis. However, the coefficients and thresholds can play a vital role in catching the change in spleen size at an early stage. Despite geometrical limitations (actual round shape of the spleen vs coefficients counted as cuboid figures), all coefficients represented a high level of correlation with the real volume of the spleen and can be used as a supportive tool in the daily clinical practice.

\section{Conclusion}

Due to the poor accuracy of splenic index, it should be replaced with:

(a) three-dimensional coefficient calculated as:

maximal length $\times$ hilum thickness $\times$ vertical height,

which establishes the strongest correlation with real spleen volume and could be assessed in order to objectively monitor spleen volume.

(b) or two-dimensional coefficient, resulting from equation:

maximal length $\times$ vertical height,

which simultaneously correlates well with the real spleen volume and has the highest values of sensitivity and specificity for the threshold of 115 , which makes it a great tool for quick splenomegaly screening in CT examination.

Acknowledgements We would like to express our gratitude to Prof. Andrzej Urbanik, MD, PhD; Anna Gajdosz and Maria Widomska for content-related support. Without their invaluable help our work would remain uncompleted.

Compliance with ethical standards

Funding None.

Conflict of interest The authors declare that they have no conflict of interest.

Ethical approval All procedures performed in studies involving human participants were in accordance with the ethical standards of the institutional research committee and with the 1964 Helsinki declaration and its later amendments or comparable ethical standards. For this type of study, formal consent is not required. This article does not contain any studies with animals performed by any of the authors.

Open Access This article is distributed under the terms of the Creative Commons Attribution 4.0 International License (http://creativecommons. org/licenses/by/4.0/), which permits unrestricted use, distribution, and reproduction in any medium, provided you give appropriate credit to the original author(s) and the source, provide a link to the Creative Commons license, and indicate if changes were made.

\section{References}

1. Wu WC, Chiou YY, Hung HH, et al. (2012) Prognostic significance of computed tomography scan-derived splenic volume in hepatocellular carcinoma treated with radiofrequency ablation. J Clin Gastroenterol 46:789-795. https://doi.org/10.1097/MCG.0b013e3 1825 ceeb5 
2. Strijk SP, Wagener DJ, Bogman MJ, de Pauw BE, Wobbes T (1985) The spleen in Hodgkin disease: diagnostic value of CT. Radiology 154:753-757. https://doi.org/10.1148/radiology.154.3. 3969481

3. Brown NF, Marks DJ, Smith PJ, Bloom SL (2011) Splenomegaly. Br J Hosp Med (Lond) 72:M166-169. https://doi.org/10.12968/ hmed.2011.72.sup11.m166

4. Grover SA, Barkun AN, Sackett DL (1993) The rational clinical examination. Does this patient have splenomegaly? JAMA 270:2218-2221. https://doi.org/10.1001/jama.270.18.2218

5. Rabushka LS, Kawashima A, Fishman EK (1994) Imaging of the spleen: CT with supplemental MR examination. Radiographics 14:307-332. https://doi.org/10.1148/radiographics.14.2.8190956

6. Prassopoulos P, Daskalogiannaki M, Raissaki M, Hatjidakis A, Gourtsoyiannis N (1997) Determination of normal splenic volume on computed tomography in relation to age, gender and body habitus. Eur Radiol 7:246-248. https://doi.org/10.1007/s00330005 0145

7. Linguraru MG, Sandberg JK, Jones EC, Summers RM (2013) Assessing splenomegaly: automated volumetric analysis of the spleen. Acad Radiol 20:675-684. https://doi.org/10.1016/j.acra. 2013.01.011

8. Lamb PM, Lund A, Kanagasabay RR, et al. (2002) Spleen size: how well do linear ultrasound measurements correlate with threedimensional CT volume assessments? Br J Radiol 75:573-577. https://doi.org/10.1259/bjr.75.895.750573

9. Elstein D, Hadas-Halpern I, Azuri Y, et al. (1997) Accuracy of ultrasonography in assessing spleen and liver size in patients with Gaucher disease: comparison to computed tomographic measurements. J Ultrasound Med 16:209-211. https://doi.org/10.7863/ jum.1997.16.3.209

10. Chen XL, Chen TW, Li ZL, et al. (2013) Spleen size measured on enhanced MRI for quantitatively staging liver fibrosis in minipigs.
J Magn Reson Imaging 38:540-547. https://doi.org/10.1002/ jmri.24007

11. Yetter EM, Acosta KB, Olson MC, Blundell K (2003) Estimating splenic volume: sonographic measurements correlated with helical CT determination. AJR Am J Roentgenol 181:1615-1620. https://doi.org/10.2214/ajr.181.6.1811615

12. Prassopoulos P, Cavouras D (1994) CT assessment of normal splenic size in children. Acta Radiol 35:152-154. https://doi.org/ $10.1177 / 028418519403500210$

13. Ishibashi H, Higuchi N, Shimamura R, et al. (1991) Sonographic assessment and grading of spleen size. J Clin Ultrasound 19:21-25. https://doi.org/10.1002/jcu.1870190106

14. Lackner K, Brecht G, Janson R, et al. (1980) The value of computer tomography in the staging of primary lymph node neoplasms (author's transl). Rofo 132:21-30. https://doi.org/10.1055/s-20081056518

15. Linguraru MG, Sandberg JK, Li Z, Pura JA, Summers RM (2009) Atlas-based automated segmentation of spleen and liver using adaptive enhancement estimation. Med Image Comput Comput Assist Interv 5762:1001-1008. https://doi.org/10.1007/978-3-642-04271-3 121

16. Bezerra AS, D'Ippolito G, Faintuch S, Szejnfeld J, Āhmed M (2005) Determination of splenomegaly by CT: is there a place for a single measurement? AJR Am J Roentgenol 184:1510-1513. https://doi.org/10.2214/ajr.184.5.01841510

17. Andrews MW (2000) Ultrasound of the spleen. World J Surg 24:183-187. https://doi.org/10.1007/s002689910031

18. Cerri GG (1985) Baço. In: Rocha DC, Cerri GG, Prando A (eds) Ultrasonografia abdominal (in Portuguese). São Paulo: Sarvier, pp 71-80

19. Elsayes KM, Narra VR, Mukundan G, et al. (2005) MR imaging of the spleen: spectrum of abnormalities. Radiographics 25:967-982. https://doi.org/10.1148/rg.254045154

20. DeLand FH (1970) Normal spleen size. Radiology 97:589-592. https://doi.org/10.1148/97.3.589 